\title{
Modeling the Perceptions and Preferences of Pedestrians on Crossing Facilities
}

\author{
Hongwei Guo, ${ }^{1}$ Facheng Zhao, ${ }^{1}$ Wuhong Wang, ${ }^{1}$ Yanlong Zhou, \\ Yujie Zhang, ${ }^{1}$ and Geert Wets ${ }^{2}$ \\ ${ }^{1}$ Department of Transportation Engineering, Beijing Institute of Technology, Beijing 100081, China \\ ${ }^{2}$ Transportation Research Institute (IMOB), Hasselt University, Wetenschapspark 5 bus 6, 3590 Diepenbeek, Belgium
}

Correspondence should be addressed to Wuhong Wang; wangwuhong@bit.edu.cn

Received 17 November 2013; Accepted 16 January 2014; Published 6 March 2014

Academic Editor: Huimin Niu

Copyright (C) 2014 Hongwei Guo et al. This is an open access article distributed under the Creative Commons Attribution License, which permits unrestricted use, distribution, and reproduction in any medium, provided the original work is properly cited.

Pedestrian's street-crossing behaviour has a significant effect on traffic performance and safety. The crossing behaviour is determined by human factors and environmental factors. Aiming at examining the pedestrian perceptions toward crossing facilities and preferences for crossing locations, an observational study of pedestrian crossing behaviour at urban street is conducted. The perceptions and preferences of pedestrians are collected using stated preference technique. A specific questionnaire is designed to conduct the stated preference survey. A multinomial logit model is proposed to describe the perceptions and preferences of pedestrians on crossing facilities and locations. The sensitivity analysis is performed to discuss the influence of various factors on crossing behaviour. Then the relationship between crossing locations and crossing distances is analyzed by a new proposed method. With the theoretical analysis, the engineering solutions considering pedestrian behaviour are suggested. The results are helpful to design human-centered crossing facilities in urban traffic.

\section{Introduction}

Pedestrians' traffic behaviour at urban network includes walk along streets and cross streets. Once pedestrians want to cross streets, it is inevitable that they will conflict with motor vehicles. Traffic accidents that involved pedestrians and cyclists have become a critical safety problem all over the world [1]. In a developing country, like China, with large population and weak infrastructure, pedestrians often become a reason for traffic congestion and traffic accidents. To deal with pedestrian traffic problems, various crossing facilities are designed to assist pedestrian in crossing safely, for example, crosswalk (signalized and unsignalized), pedestrian overpass, and pedestrian underpass at intersection or midblock. With crossing facilities, pedestrians are separated from motor vehicles temporally or spatially. Unfortunately, pedestrians' crossing behaviour is strongly related to human factors. Thus pedestrians may cross illegally rather than using crossing facilities. The subjectivity and randomness make pedestrian behaviour complicated and also encourage traffic engineers to pay more attention to pedestrian traffic.
The research on pedestrian characteristics is the basic and important part for traffic engineering. Fruin [2] published the monograph Pedestrian Planning and Design and it is regarded as a foundation for pedestrian research. Then some particular researches included studies for behaviour, psychology, safety, and simulation. Mohammed [3] evaluated pedestrian crossing speed in Jordan and evaluated the effect of age, gender, and distance crossed (street width). Lam et al. [4] studied the relationship between walking speed and pedestrian flow under various flow conditions and the effects of bidirectional pedestrian flow on signalized crosswalks in Hong Kong. Sisiopiku and Akin [5] presented findings from an observational study of pedestrian behaviour at various urban crosswalks in a divided urban street near university campus. Pedestrian level-of-service assessment models were introduced by different methods under various traffic conditions, and standards for each level were estimated $[6,7]$.

Pedestrian behaviour is very complex and easily influenced by environmental designs and urban forms. A proper design of facilities can encourage walking without compromising safety and convenience $[8,9]$. The waiting time 


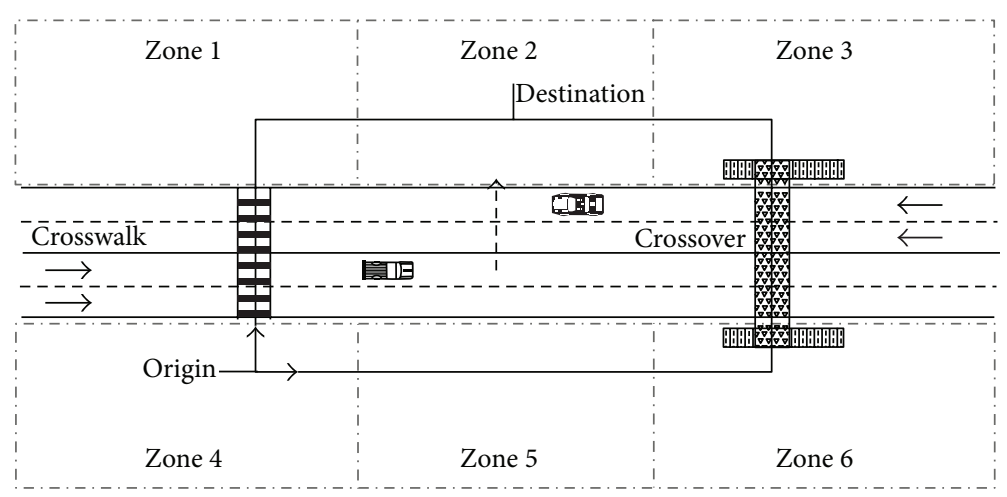

FIGURE 1: Illustration of crossing route.

and crossing distance (distance between trip destination and actual crossing location) are the mainly external factors which would lead to unsafe crossing. The need to hurry or the desire to keep moving along the shortcut is the main subjective reason behind the lack of compliance with pedestrian signals or crossing facilities. Pedestrian violation can be considered as the inevitable outcome of the contradiction between external factors and human factors. $\mathrm{Li}$ [10] and Lambrianidou et al. [11] gave various reports about pedestrians' behaviour influenced by time and distance. Guo et al. $[12,13]$ analysed the waiting behaviour during the street crossing by using the reliability theory and indicated the violation risk quantitatively with the increasing waiting time. Chu et al. [14] used data obtained from pedestrians' stated crossing preference and explained the stated preference with the street environment within the framework of disaggregate models. Yannis et al. [15] improved Chu's model to evaluate accident risk along a trip in relation to the estimated crossing behaviour of pedestrians. Nassiri and Sajed [16] evaluated and identified the effective parameters in pedestrian's decisionmaking process based upon vehicle speed and headway on multilane streets by using logit model.

It comes to a conclusion that a series of studies have provided insight into several aspects of pedestrian crossing behaviour. Compared with the researches about pedestrian accident analysis and safety evaluation, the researches about the preferences and perceptions of pedestrians on crossing facilities and crossing locations are limited. While crossing a street, the crossing facilities and crossing locations play an important role. The existence of crossing facilities is to ensure safety and assist accessibility. However, some pedestrians do not like using crossing facilities and even cross street illegally because they do not think the facilities can meet their demands. This paper aims at evaluating pedestrian preferences for commonly encountered crossing facilities and analysing pedestrian crossing locations in different conditions. To achieve such objective, a multinomial logit model for pedestrian crossing behaviour is proposed to analyse the probability distribution of utilities for various crossing facilities. With the evaluated model, pedestrian crossing locations are examined in various assumed traffic conditions so as to explore the reasons behind the crossing behaviour. This paper hopes to give a better understanding of pedestrian crossing behaviour.

The paper is organized as follows. Section 2 introduces the methodology including discrete choice model and data collection. Section 3 describes the model demonstration and estimation. Section 4 discussed pedestrian crossing behaviour under various influential factors. Section 5 presents some engineering improvement for pedestrian facilities considering the crossing behaviour. Section 6 presents some conclusions from this study and a tentative plan for future work.

\section{Methodology}

2.1. Analysis of Pedestrian Crossing Behaviour. As mentioned above, pedestrian crossing behaviour is strongly related to human factors and traffic circumstances. Pedestrians' decision making about where to cross or when to cross can be described as the process of perception-judgment-decisionaction. Generally, crossing decision is influenced by characteristics of the trip (e.g., the origin and destination and the complexity and the length of the route), characteristics of the infrastructure (e.g., the type of pedestrian facility, road geometry, and traffic conditions), and individual characteristics (e.g., age and gender and safety awareness). The crossing behaviour shall therefore reflect the combined assessment of the above characteristics under all sorts of conditions. In accordance with human nature, crossing behaviour exhibits significant subjectivity and randomness. Therefore, pedestrian crossing behaviour may become risk-taking action and lead to conflicts with motor vehicles.

The process of street crossing can be explained by the utility maximization theory that pedestrians want to choose the most satisfactory facilities and locations to cross the street. As a result, pedestrians get the maximum utility. It would be reasonable to assume that pedestrians' most satisfactory decisions are dependent on the location and the type of crossing facility. For example, as shown in Figure 1, there are two crossing facilities in the area. If a pedestrian's origin is in zone 5 and the destination is in zone 2, there are three potential routes to cross the street. The first route is to use 
the crosswalk, the second route is to use the overpass, and the third route is to cross at any location. Therefore the existence of crossing facility may change pedestrians' crossing behaviour and also induce traffic violation because pedestrians cannot cross the street at the desired location.

2.2. Discrete Choice Model Framework. The utilitarian approach of microeconomic concepts is employed and the discrete choice model framework is adopted to describe crossing behaviour. The discrete choice model is one of the most important models for the research on traffic behaviour and is widely used in transportation predictions [17]. According to the stochastic utility model, various alternatives have utilities which will influence the choice. In the behaviour of facility choice, the alternative $i$ (i.e., the crossing facility i) within set of all elemental alternatives $A_{I}$; the utility function $U_{\text {in }}$ of facility $i$ selected by pedestrian $n$ consists of an observable utility component $V_{\text {in }}$ and the unobservable random component $\varepsilon_{i n}$. The utility can be defined as

$$
U_{\text {in }}=V_{\text {in }}+\varepsilon_{\text {in }} \quad \forall i \in A_{I} ; i=1, \ldots, I ; n=1, \ldots, N \text {. }
$$

Assuming that each pedestrian is the decision maker of him/herself, the selection criterion is to maximize the utility. In the stochastic utility model, the probability to choose crossing facility $n$ is

$$
\begin{aligned}
P_{\text {in }} & =\operatorname{Prob}\left(U_{\text {in }} \geq \max U_{k n} ; i \neq k, k \in A_{I}\right) \\
& =\operatorname{Prob}\left(V_{\text {in }}+\varepsilon_{\text {in }} \geq \max \left(V_{k n}+\varepsilon_{k n}\right)\right),
\end{aligned}
$$

where $P_{\text {in }}$ is the probability of crossing facility $i$ selected by pedestrian $n, U_{k n}$ is the utility function of other alternatives in choice set $A_{I}$ excluding the alternative $i$, and $\operatorname{Prob}(*)$ is the probability function.

Operational models are based on specific assumptions about the distribution of $\varepsilon_{i n}$. Assuming i.i.d. extreme value distributions lead to the multinomial logit (MNL) model, which has been very successful due to its computational and analytical tractability. The MNL model for the selection behaviour of pedestrian is defined as

$$
P_{i n}=\frac{\exp \left(V_{i n}\right)}{\sum_{j \in A_{I}} \exp \left(V_{j n}\right)} .
$$

2.3. Data Collection. Data collection was taken in specific area with more than two types of crossing facilities. The spacing between two facilities was within 300 meters. Moreover, there were large pedestrians to cross the street. The survey area was divided into 6 zones (or 8 zones) so that the pedestrians' origins and destinations can be recorded accurately. Finally, the Zhongguancun Street and Xidan Street in Beijing were chosen as survey areas.

The data collection contained field survey and questionnaire survey. The field survey obtained the information about pedestrians crossing behaviour. The field survey was conducted by observers at the appointed site and it contained two aspects: (a) to observe the crossing locations of pedestrians who had participated in the questionnaire survey and (b) to observe and record the amounts of pedestrians used crossing facilities. Questionnaire survey was conducted to collect stated preference (SP) data. The design of the questionnaire should meet preset criteria such as inclusion of the statement of the study purpose and importance, clear definition of questions, and avoidance of personal or potentially offensive questions. The contents of questionnaire contained (a) pedestrians' personal profile (age and gender), (b) preference for crossing facilities (crossing location, detour distance, and compliance conditions), (c) action principle of crossing choice (i.e., which one is considered to be the first importance: safety, convenience, saving time, or saving strength?), (d) jaywalking conditions (i.e., reason and frequency), and (e) attitude towards detour (i.e., acceptance level and acceptable distance). The questionnaire was pretested before real survey. The whole survey for each pedestrian was finished in real traffic condition and it took less than 2-3 minutes. The information about the data collection and corresponding definition of variables in the survey are shown in Table 1.

At the end of the survey, 402 available questionnaires were received. There were 205 male pedestrians and 197 female pedestrians participating in the survey. The most participants were under 40 years of age. In the field survey, 1158 pedestrians' crossing locations and routes were recorded. These records also included the pedestrians who participated in the questionnaire survey. From the survey results, a majority of pedestrians used overpass/underpass to cross (46.4\% used overpass, $32.7 \%$ used crosswalk, and $20.9 \%$ jaywalked) and most of them stated that they preferred overpass/underpass in questionnaire $(42.1 \%$ choose overpass and $22.3 \%$ choose underpass). The remaining $35.6 \%$ preferred to cross at pedestrian crosswalk. About the action principle of crossing choice, $50.49 \%$ of participants thought of safety as the principle of crossing, $32.17 \%$ chose convenience, and $17.32 \%$ chose saving time or physical strength. The attitude towards the detour indicated that $30.6 \%$ of participants were willing to detour to use the crossing facility and $6.7 \%$ of them reject to detour. The remaining pedestrians stated they would accept detour in certain conditions (i.e., 37.3\% of them detour sometime and $25.5 \%$ of them detour occasionally). Other results showed that most pedestrians (86.13\%) could accept a detour distance less than 100 meters. And the results indicated that people are willing to detour in subjective desire but the detour distance they can accept is short relatively.

The survey results show the pedestrians' preference for crossing facilities. And the SP data are basically accordant with the RP data so that a conclusion for the survey can be drawn: the survey is available.

\section{Model Demonstration and Estimation}

3.1. Model Demonstration. According to the stochastic utility theory, the alternatives have various utilities that will influence the choice. In this paper, three alternatives are considered, including pedestrian crosswalk, overpass (underpass), and jaywalking. The overpass and underpass have the similar physical structure and safeguard so they are classified as the same alternative. Jaywalking means a pedestrian does 
TABLE 1: Definition of variables.

\begin{tabular}{|c|c|c|}
\hline Variable & Definition & Note \\
\hline$X_{n 1}$ & Age & Age group: (1) $15-23,(2) 24-30$, , (3) $31-40,(4) 41-55$, and (5) $>55$ \\
\hline$X_{n 2}$ & Gender & Pedestrian's gender: (1)—male and (2)—female \\
\hline$X_{n 3}$ & Principles of crossing & $\begin{array}{l}\text { The principles of street crossing: (1) safety, (2) convenience, and (3) save time or } \\
\text { strength }\end{array}$ \\
\hline$X_{n 4}$ & Detour willingness $^{\mathrm{a}}$ & $\begin{array}{l}\text { Pedestrian's attitude towards detour: (1) accept to detour, (2) often detour, (3) } \\
\text { occasionally detour, and (4) refuse to detour }\end{array}$ \\
\hline$X_{n 5}$ & Compliance with the traffic rules & The degree of compliance with the traffic rules: (1) always, (2) often, and (3) seldom \\
\hline$X_{n 6}$ & Crossing time & $\begin{array}{l}\text { The time for crossing at crosswalk: (1) pedestrian green signal, (2) pedestrian red } \\
\text { signal and force to cross, and ( } 3 \text { ) pedestrian red signal and the road is clear }\end{array}$ \\
\hline$X_{n 7}$ & Illegal reason & $\begin{array}{l}\text { The reason behind the illegal crossing: (1) unreasonable design and (2) pedestrians' } \\
\text { subjective reasons }\end{array}$ \\
\hline$X_{n 8}$ & Conformity psychology & (1) Pedestrians follow others to cross street (2) Pedestrians do not follow others \\
\hline$X_{n 9}$ & Detour distance & The additional distance caused by detour \\
\hline$X_{n 10}$ & Travel time & The time spent on crossing street via crossing facility \\
\hline$X_{n 11}$ & Origin and destination & The origin and destination of the trip of a pedestrian \\
\hline
\end{tabular}

TABle 2: Model demonstration.

\begin{tabular}{lccc}
\hline \multirow{2}{*}{ Variable } & \multicolumn{3}{c}{ Alternative } \\
& Overpass/underpass & Crosswalk & Jaywalking \\
\hline constant & & $\bullet$ & $\bullet$ \\
$X_{n 1}$ & $\bullet$ & & \\
$X_{n 2}$ & & $\bullet$ & \\
$X_{n 3}$ & $\bullet$ & & $\bullet$ \\
$X_{n 4}$ & $\bullet$ & & \\
$X_{n 5}$ & & $\bullet$ & $\bullet$ \\
$X_{n 6}$ & & & \\
$X_{n 7}$ & & $\bullet$ & $\bullet$ \\
$X_{n 8}$ & & & $\bullet$ \\
$X_{n 9}$ & $\bullet$ & $\bullet$ & $\bullet$ \\
$X_{n 10}$ & $\bullet$ & $\bullet$ & \\
$X_{n 11}$ & & & \\
\hline
\end{tabular}

not cross the street using crossing facilities. Additionally, the model is based on the hypotheses that the pedestrian himself is the decision maker of crossing the street and he always chooses the satisfactory facilities. With the alternatives and explanatory variables adopted above, utility functions for each alternative can be defined. The variables and components of utility functions are shown in Table 2.

3.2. Model Evaluation. With the model evaluation, the parameters for the explanatory variables are estimated in each utility function by fitting the models to the observed choice data. Maximum likelihood estimation and $t$-test are adopted.
When the absolute value of $t$-test of one explanatory variable is greater than 1.96, this variable will be considered that it can influence the choice with the probability of $95 \%$. If there is any variable that cannot influence the result significantly, it will be excluded and the other parameters will be estimated again. From initial estimation, the pedestrian's gender (variable $X_{n 2}$ ) and pedestrian's compliance with the traffic rules (variable $X_{n 6}$ ) are rejected. Then the remainder variables are estimated again and the results are shown in Table 3. With the results, a conclusion can be drawn that the model is well behaved. First, all explanatory variables can influence the choice significantly. Second, the variables fit the data well that the $\rho^{2}$ adjusted for the number of variables is 0.396 . In contrast, it is common to see an adjusted $\rho^{2}$ below 0.3 in discrete choice model such as mode choice models [14].

3.3. Validating Model Credibility. The estimated model is applied with survey data to compare with the actual observations to establish the credibility of choice models and to evaluate whether or not the model demonstrates real behaviour. The alternative with maximum probability will be chosen. If a calculation result is accordant with the survey data, this is defined as a hit result. A hit result means a pedestrian's behaviour is modeled exactly. Let $S_{\text {in }}$ represent the hit result that pedestrian $n$ selects facility $i$. Consider the following equation:

$$
S_{i n}= \begin{cases}1 ; & \begin{array}{l}
\text { if the calculated result is equal to } \\
\text { the survey result }
\end{array} \\
0 ; & \text { otherwise. }\end{cases}
$$


TABLE 3: Coefficient estimation for explanatory variable.

\begin{tabular}{lcccccccc}
\hline Coefficient & $X_{n 1}$ & $X_{n 3}$ & $X_{n 4}$ & $X_{n 5}$ & $X_{n 6}$ & $X_{n 7}$ & $X_{n 8}$ \\
\hline Estimation & 0.9112 & -0.5828 & -0.906 & -0.4792 & -2.341 & -0.7021 & -0.014 & -0.0523 \\
$t$-test & -2.3632 & 7.8355 & -4.3234 & -2.3632 & -4.6136 & 4.2314 & -2.8926 & -2.2793 \\
\hline
\end{tabular}

TABLE 4: Hit ratio of MNL model.

\begin{tabular}{lcccc}
\hline Alternative & Observed result & Calculation result & Accordant result & Hit ratio (\%) \\
\hline Crosswalk & 131 & 131 & 97 & $74.05 \%$ \\
Overpass & 187 & 187 & 154 & $82.44 \%$ \\
Jaywalk & 84 & 84 & 59 & $70.23 \%$ \\
\hline Total & 402 & 402 & 310 & $77.11 \%$ \\
\hline
\end{tabular}

The hit ratio can be calculated as

$$
\begin{gathered}
R_{i}=\sum_{i=1}^{3} \frac{S_{i n}}{N_{i}}, \\
R=\sum_{i=1}^{3} \frac{\sum_{i \in J_{m}} S_{i n}}{\sum_{m=1}^{3} J_{m}},
\end{gathered}
$$

where $R_{i}$ is hit ratio of alternative $i, R$ is hit ratio of all alternatives, $N_{i}$ is the number of samples belonging to alternative $i$, and $J_{m}$ is the number of entire samples.

The results of the hit ratio can be seen in Table 4 .

From the results in Table 4 , the hit ratio has indicated that the model can explain the preference of crossing facilities preferably. With the proposed model, the crossing behaviour can be discussed in detail.

\section{Discussion of Pedestrian Crossing Behaviour}

With the aim of analyzing the crossing behaviour at various pedestrian facilities, pedestrians' crossing behaviour is discussed under specific hypothetical conditions. Here, sensitive analysis is performed using the proposed model to illustrate the impact of the considered variables, for example, action principles, detour willingness, and distance. These analyses are important to understand pedestrian behaviour.

4.1. Effect of Action Principle. When pedestrians want to cross the street, the action principle will influence the crossing choice significantly. If pedestrians consider safety the first importance while crossing street, they probably choose crossing facilities instead of illegal crossing. On the other hand, if the convenience is considered the first importance, jaywalking is easy to happen. This phenomenon is verified by the survey data: $41 \%$ of the samples consider safety the first importance and 39\% have chosen the overpass/underpass. In order to study the influence of the action principle, three hypotheses are used: (a) all pedestrians choose safety as action principle, (b) convenience is chosen, and (c) saving time or physical strength is chosen. Pedestrians' crossing choices are evaluated by MNL model and the results are shown in Figure 2. In the results of hypothesis (a),

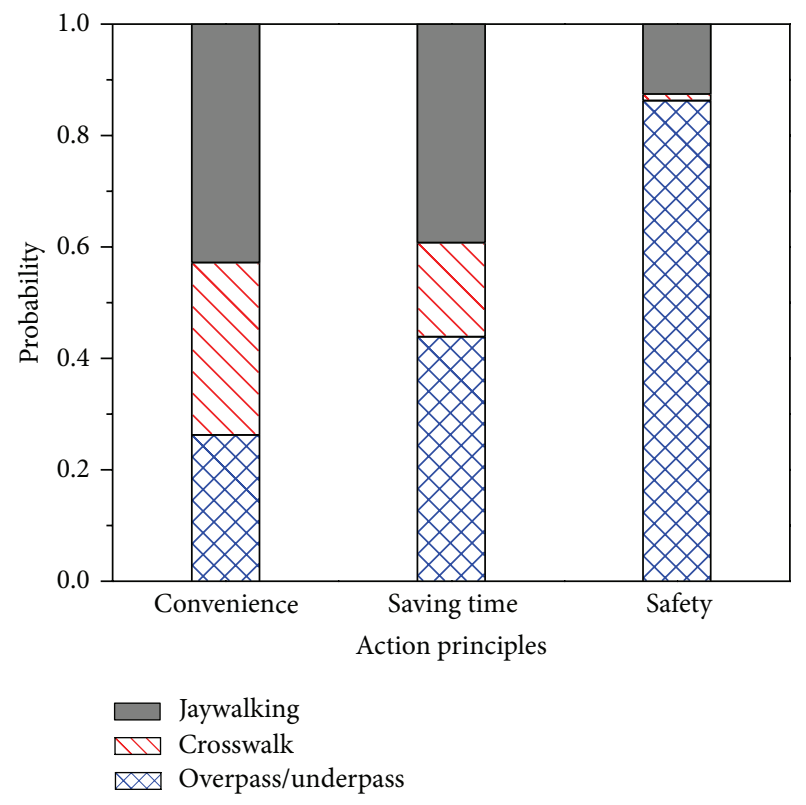

FIGURE 2: Probability of each alternative with various action principles.

the overwhelming majority of pedestrians (86.0\%) will choose overpass/underpass to cross the street and only $1.2 \%$ of pedestrians will choose crosswalk. The results agree with the truth that overpass/overpass can provide better safeguard for pedestrians. Additionally, there are still a certain number of jaywalkers $(12.8 \%)$ but this is not contrary to the model: not all pedestrians want to use crossing facilities. In the results of hypothesis (b), convenience is the action principle and the results show that almost half of the pedestrians (43.6\%) will cross illegally. This is accordant with the current situation that jaywalking may be more convenient than crossing at specified facilities. Sometimes, using pedestrian facilities means increasing walking distance or waiting time. Pedestrians who choose the crosswalks (31.6\%) are more than people who choose the overpass/underpasses (26.8\%). The results of hypothesis (b) are similar to those of hypothesis (c). Jaywalking may be a proper approach to saving time or strength to a certain degree, although it is illegal. 


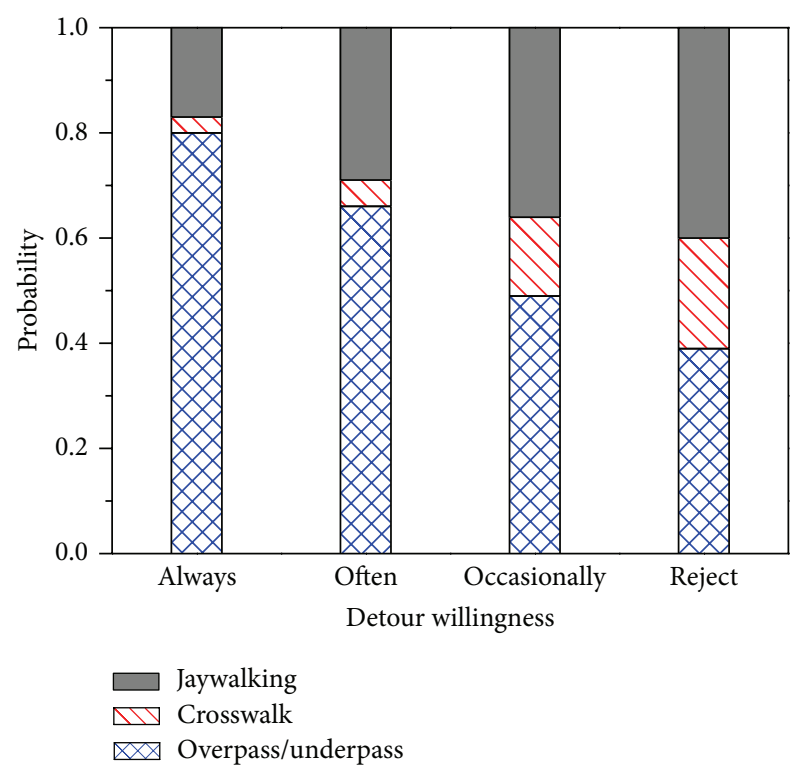

Figure 3: Probability of each alternative with different detour willingness.

4.2. Effect of Detour Willingness and Detour Distance. In real traffic condition, pedestrians may detour to arrive at the location with crossing facilities rather than crossing anywhere or anytime they want. Such detour increase crossing distance and influence crossing behaviour significantly. Therefore, it is important for engineers to design crossing facilities with reasonable interval distance. Here, pedestrians' attitudes about the detour and acceptable detour distance are considered. According to the questionnaire survey, pedestrians' attitudes towards the detour are evaluated by the frequency of detour, which is divided into four levels: always accept, often, occasionally, and reject. Here the crossing behaviour is represented under assumed conditions if all of the pedestrians select the same attitude to cross the street. From the results, as shown in Figure 3, with decrease of the acceptance frequency, the probability that the overpass/underpass is chosen is in a downtrend. In contrast, the probabilities that the crosswalk or jaywalking is chosen increases and the probability of jaywalking would increase even faster. These results are accordant with the reality that jaywalking is a direct and convenient way if pedestrians are not willing to detour to use the crossing facilities. Furthermore, the survey data also confirm the result (only $30.6 \%$ of the participants can accept detour and nearly half of them seldom detour).

On the other hand, the acceptable distance for detouring is important to explain the crossing behaviour that people cannot cross at the expected location. Pedestrians' attitudes towards detour would reflect their personal desire, but the detour distance may change the final choice. The survey data present the fact that majority of participants $(86.13 \%)$ are willing to accept the detour distance within 100 meters. It means, if the detour distance is longer than 100 meters, pedestrians may change their mind even jaywalk. With the help of MNL model, the sensitivity about detour distance is analyzed. As shown in Figure 4, it is clear that the detour distance can influence the probability of jaywalking.

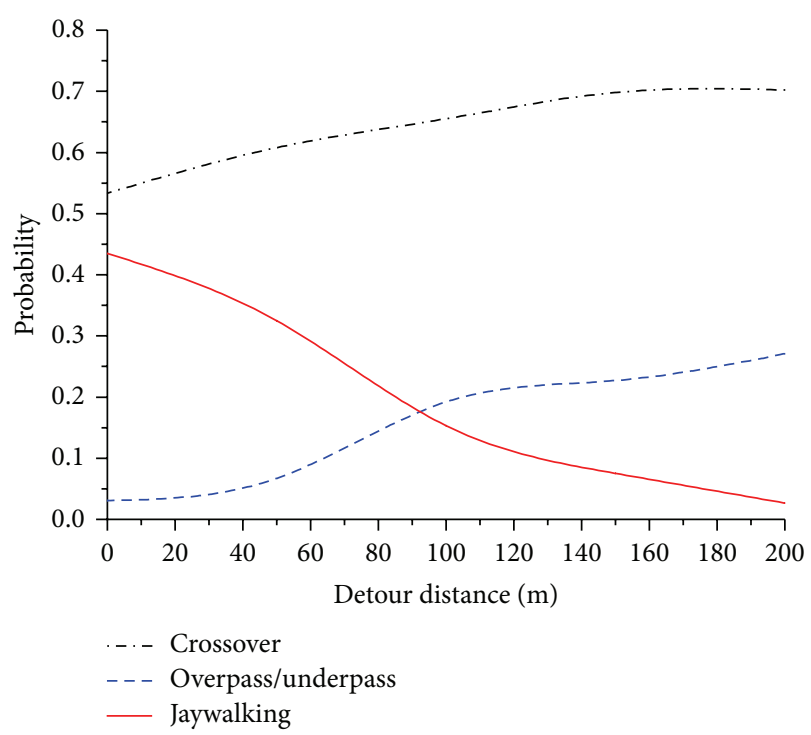

Figure 4: Probability of each alternative with different acceptable detour distance.

The results show that only $2.74 \%$ of people will cross illegally on the premise that acceptable distance is 200 meters. On the contrary, $43.5 \%$ of pedestrians may jaywalk if such distance is shorter than 50 meters.

Here, a parameter named compliance index (CI) is defined as the difference that the probability of legal crossing minus jaywalking probability. This parameter indicates the level to which pedestrians comply with traffic regulations when they are in conditions of various detour distances. So larger CI parameter means only a few pedestrians violate the traffic regulations. As shown in Figure 5, the CI curve decreases with the increase of detour distance. A rapid decrease appears between 75 and 150 meters. This trend is accordant with the survey results and it is useful to give some suggestions for design of pedestrian facilities. According to the curve fitting, the function of $\mathrm{CI}$ is given by

$$
D_{i}(S)=\alpha \cdot \tanh \left(\frac{s}{\varphi}-\gamma\right)+\beta,
$$

where $D_{i}(S)$ is function of CI at location $i$ and $S$ is detour distance to get location $i$.

The CI curve is shown as a blue line and the fitting curve of (6) (with $\alpha=0.38, \beta=0.45, \varphi=46.66$, and $\gamma=2.45$ ) is shown as a red line in Figure 5. As shown in Figure 5, (6) has a fair goodness of fit and it can present the features of CI curve.

4.3. Walk First or Cross First. The above discussions about detour focus on the scene that the destination is opposite to the origin. On the other hand, the destination is on the other side of the street diagonally and there are several crossing facilities located between them. In this scene, pedestrians would consider whether cross first or walk first. For example, the origin is in zone 1 and the destination is in zone 6 (as shown in Figure 1), there are two choices: to cross first via 


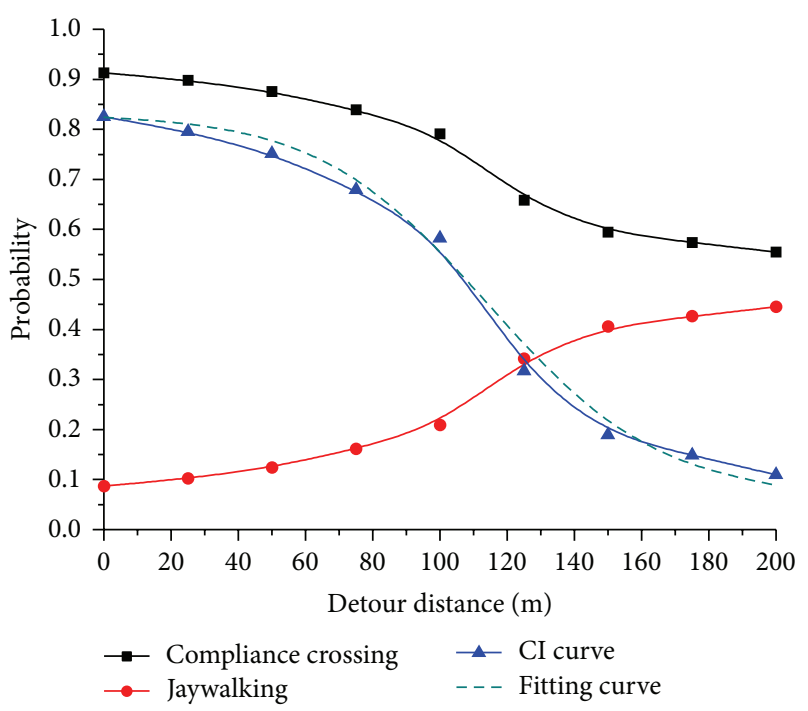

FIGURE 5: Illustration of compliance index.

the crosswalk or walk first then cross via the overpass. On the basis of the survey, the probabilities of the above questions are given by the following.

Cross first and walk later: $P_{c}=0.632$.

Walk first and cross later: $P_{w}=0.368$.

This means pedestrians are inclined to cross earlier. The result can be explained by the fact that people may miss a proper crossing location if they keep going. Especially for people who are not familiar with the locations of crossing facilities, they are willing to cross at first when they get a cross facility. Yannis et al. [15] obtained a similar result and gave a linear formula of probability distribution to describe the cross first behaviour. In the research of Yannis et al., a pedestrian's trip may include several blocks, so people may have diverse choices such as midblock or intersection. But it is not necessary for pedestrians to walk so long distance in most real conditions. While walking along the trip, pedestrians who are familiar with the traffic environment can choose the cross locations they prefer. However, the strangers are inclined to use the first facility they get. This phenomenon gives a message that crossing facilities should be near the generating/attracting source of walk trip.

\section{Applications and Engineering Improvements}

According to the analysis of pedestrian behaviour, it is known that pedestrians' subjective willingness can determine the final choice of crossing location. The influential factors lead to diverse choices in the same circumstance: some pedestrians prefer the crossing facilities, but some cross illegally. This is because pedestrians themselves are very appropriate to identify whether the traffic environments are desirable. Reasonable designing and operation of crossing facilities which match pedestrians' characteristics can conduct the pedestrians' behaviour and increase the use of pedestrian facilities.
So the requirements in designing of crossing facilities are to satisfy pedestrian needs and improve pedestrian safety and comfort. Here, some engineering improvements are discussed based on pedestrians' behavioural characteristics in order to make contribution to the traffic environments.

(a) Grade-separated crossing facilities are recommended for pedestrian safety and traffic performance. Though these facilities are built under severe construction conditions, pedestrian overpass or underpass is the best choice, especially in business quarters and residential areas.

(b) In the sight of pedestrian, the shorter interval distance is more convenient. Considering the requirements of performance and fairness, the commendatory interval distance between two crossing facilities is 300 meters (400 meters at the most). This is because a shorter interval will increase traffic delay and decrease the utility rate of facilities. On the contrary, the long interval may increase pedestrian violation.

(c) The consideration about the location and type of crossing facilities is very important. Pedestrians' behavioural characteristics should be considered seriously, as well as the land use around the street. The main generating/attracting sources of walk trip (e.g., school and shopping mall) should be concerned specially. In other words, crossing facilities mainly serve such trip sources.

(d) In response to the situation where pedestrian needs to detour, the strategy is the nearer the better. If the detour distance is inevitable, such distance is recommended to be less than 150 meters. This scheme aims to reduce the possibility of jaywalking and it is important for the area with large pedestrian flow.

(e) If there are trip generating/attracting sources at both side of the street diagonally, pedestrians will face the alternatives whether cross first or walk first. According to above discussion, pedestrians are inclined to cross first and walk later. Therefore it will be better to set up two facilities for each source if the interval is more than 200 meters. On the other hand, if the interval is shorter than 200 meters, it will be better to design a facility in the middle of them.

Finally, to strengthen the awareness of traffic safety is the most basic and the most efficient measure to minimize pedestrian violation.

\section{Conclusions}

This paper examines the pedestrians' preferences of crossing locations and the influential factors in making a decision to cross a street. Information is obtained through questionnaire survey and field observation in Beijing. A multinomial logit model is used to describe the crossing behaviour under various traffic conditions. And how the influential factors have impact on the crossing action is revealed by sensitivity analysis. The following conclusions are drawn based upon the results. 
(a) There is a relatively good agreement between the information of questionnaire and pedestrian actual behaviour in terms of their crossing choices. The MNL model can describe crossing behaviour preferably.

(b) The most influential subjective factor in making a decision to cross at a designated crossing location is the action principle (variable $X_{n 3}$ ). The most influential factor in external environment is the detour distance (variable $X_{n 9}$ ).

(c) A reasonable designing and a proper operation of crossing facilities can conduct crossing behaviour and increase the utilization rate of facilities. Pedestrians themselves are the most appropriate group to identify the rationality and serviceability of pedestrian environment.

(d) The results indicate that pedestrians prefer overpass/underpass, and most pedestrians consider safety the first importance. In a view of cost and construction conditions, signalized crosswalk is recommended to help minimize the pedestrian-vehicle conflicts.

The findings from this paper are expected to help understand pedestrians' behaviour at crossing locations well. Though pedestrians' preferences and the reasons behind the choice are explained by a discrete choice model, there are still some insufficiencies in the work. For example, some potential influential factors may be neglected or the factor's influencing degree is weakened so that the model cannot reflect the effects of variables comprehensively. Additionally, aggregate results for crossing behaviour with various OD pairs and the reason for pedestrian violation should be considered further.

\section{Conflict of Interests}

The authors declare that there is no conflict of interests regarding the publication of this paper.

\section{Acknowledgments}

This research was supported in part by the National Nature Science Foundation of China under Grants 51378062 and 71301010 and the Introducing Talents of Discipline to Universities under Grant B12022.

\section{References}

[1] M. Huan, X. B. Yang, and B. Jia, "Crossing reliability of electric bike riders at urban intersections," Mathematical Problems in Engineering, vol. 2013, Article ID 108636, 8 pages, 2013.

[2] J. J. Fruin, Pedestrian Planning and Design, Metropolitan Association of Urban Designers and Environmental Planners, New York, NY, USA, 1971.

[3] S. T. Mohammed, "Evaluation of pedestrian speed in Jordan with investigation of some contributing factors," Journal of Safety Research, vol. 32, no. 2, pp. 229-236, 2001.
[4] W. H. K. Lam, J. Y. S. Lee, and C. Y. Cheung, "A study of the bi-directional pedestrian flow characteristics at Hong Kong signalized crosswalk facilities," Transportation, vol. 29, no. 2, pp. 169-192, 2002.

[5] V. P. Sisiopiku and D. Akin, "Pedestrian behaviors at and perceptions towards various pedestrian facilities: an examination based on observation and survey data," Transportation Research F: Traffic Psychology and Behaviour, vol. 6, no. 4, pp. 249-274, 2003.

[6] S. Sarkar, "Evaluation of safety for pedestrians at macro- and microlevels in urban areas," Transportation Research Record, no. 1502, pp. 105-118, 1995.

[7] J. Y. S. Lee, P. K. Goh, and W. H. K. Lam, "New level-ofservice standard for signalized crosswalks with bi-directional pedestrian flows," Journal of Transportation Engineering, vol. 131, no. 12, pp. 957-960, 2005.

[8] R. Elvik, M. W. J. Sørensen, and T.-O. Nævestad, "Factors influencing safety in a sample of marked pedestrian crossings selected for safety inspections in the city of Oslo," Accident Analysis and Prevention, vol. 59, pp. 64-70, 2013.

[9] K. Shriver, "Influence of environmental design on pedestrian travel behavior in four Austin neighborhoods," Transportation Research Record, no. 1578, pp. 64-75, 1997.

[10] B. Li, "A model of pedestrians' intended waiting times for street crossings at signalized intersections," Transportation Research B: Methodological, vol. 51, pp. 17-28, 2013.

[11] P. Lambrianidou, S. Basbas, and I. Politis, "Can pedestrians' crossing countdown signal timers promote green and safe mobility?" Sustainable Cities and Society, vol. 6, pp. 33-39, 2013.

[12] H. Guo, W. Wang, W. Guo, X. Jiang, and H. Bubb, "Reliability analysis of pedestrian safety crossing in urban traffic environment," Safety Science, vol. 50, no. 4, pp. 968-973, 2012.

[13] H. Guo, Z. Gao, X. Yang, and X. Jiang, "Modeling pedestrian violation behavior at signalized crosswalks in China: a hazardsbased duration approach," Traffic Injury Prevention, vol. 12, no. 1, pp. 96-103, 2011.

[14] X. Chu, M. Guttenplan, and M. R. Baltes, "Why people cross where they do: the role of street environment," Transportation Research Record, no. 1878, pp. 3-10, 2003.

[15] G. Yannis, J. Golias, and E. Papadimitriou, "Modeling crossing behavior and accident risk of pedestrians," Journal of Transportation Engineering, vol. 133, no. 11, pp. 634-644, 2007.

[16] H. Nassiri and Y. Sajed, "Using a logit model to predict pedestrian crossing behaviour based upon vehicle speed and headway on multi-lane street," in Proceedings of the 88th Annual Meeting of the Transportation Research Board, Washington DC, USA, 2009.

[17] M. Ben-Akiva and L. Steven, Discrete Choice Analysis: Theory and Application to Travel Demand, MIT Press, Cambridge, Mass, USA, 1985. 


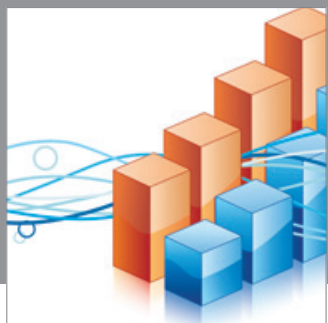

Advances in

Operations Research

mansans

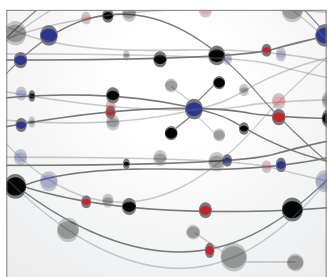

The Scientific World Journal
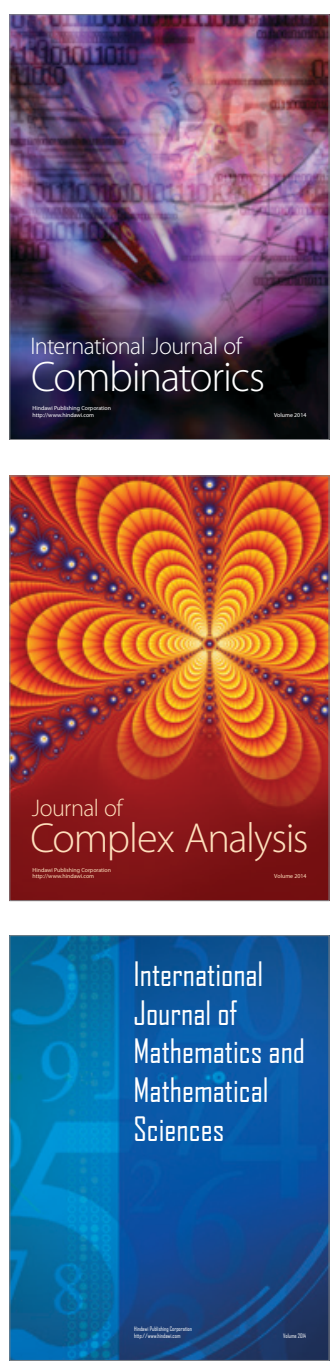
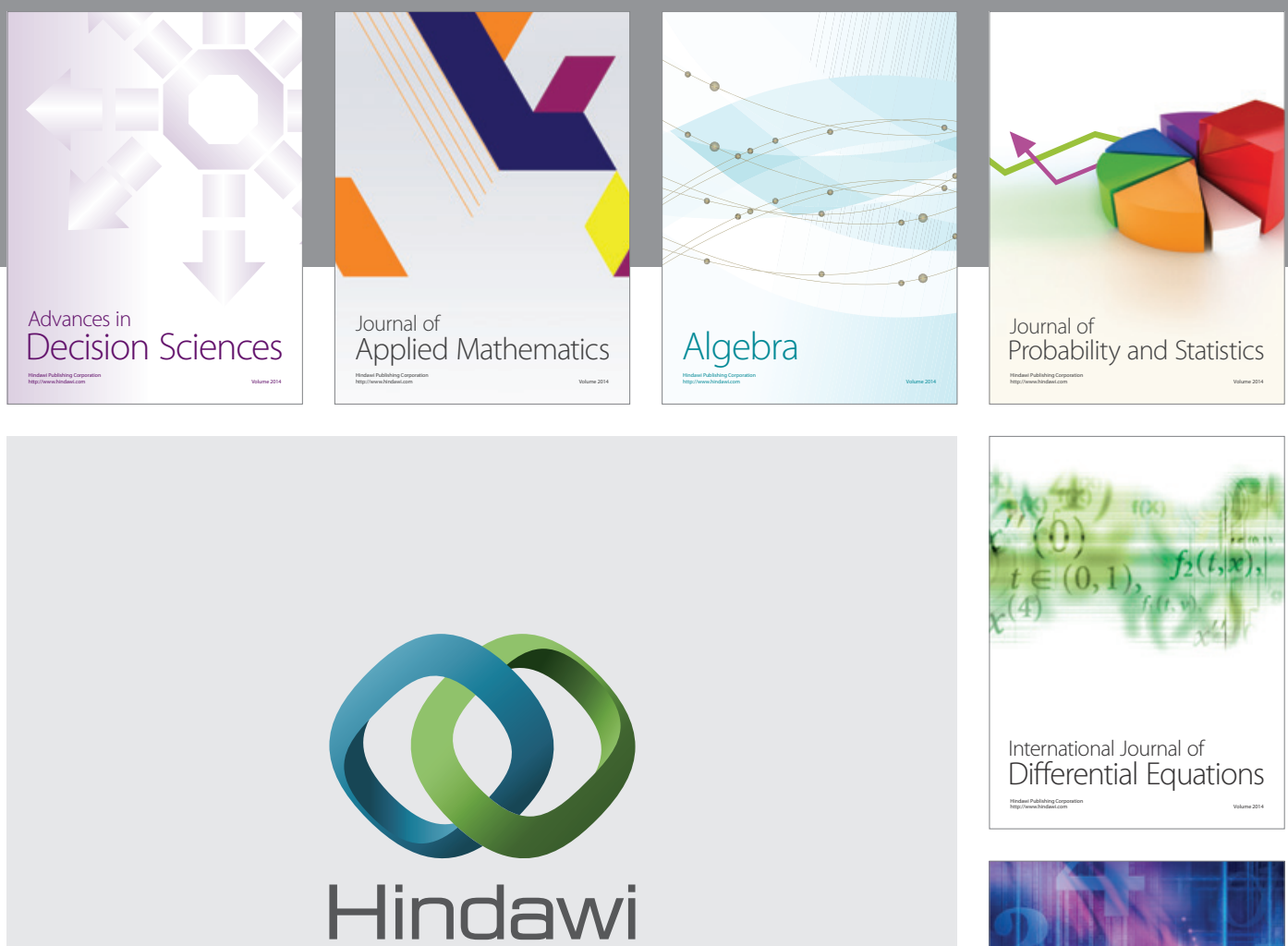

Submit your manuscripts at http://www.hindawi.com
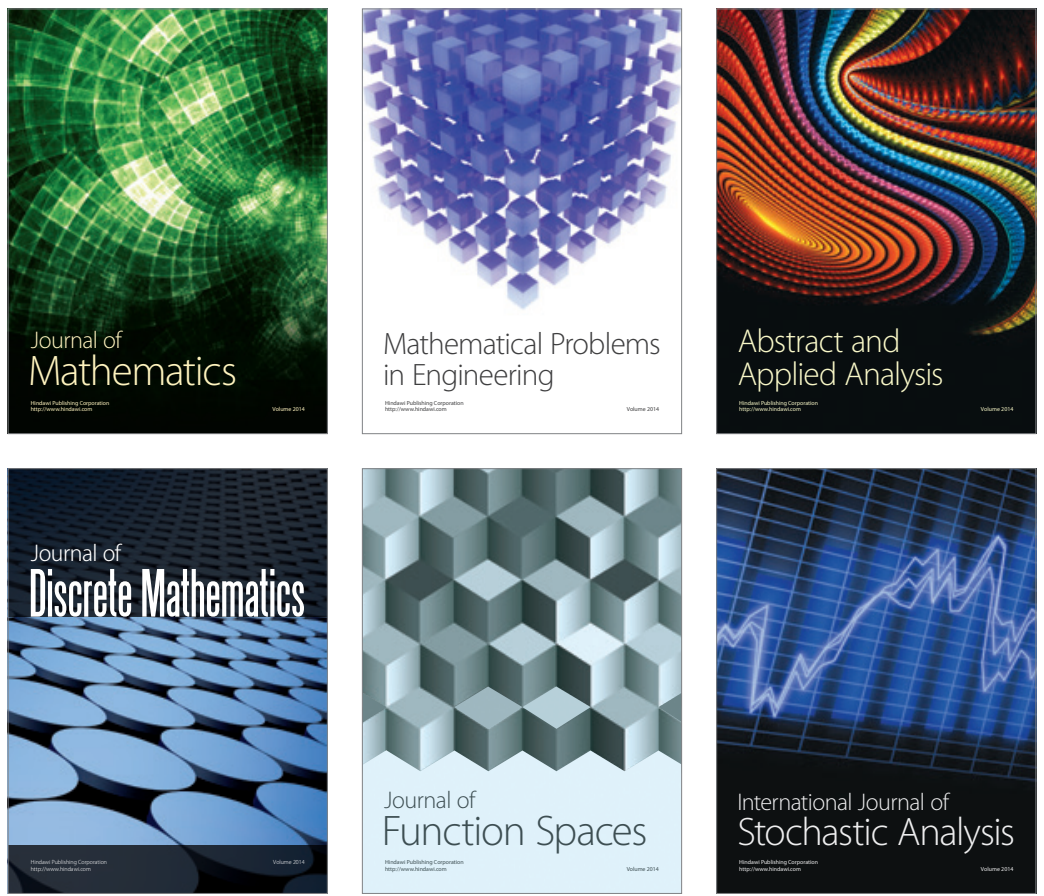

Journal of

Function Spaces

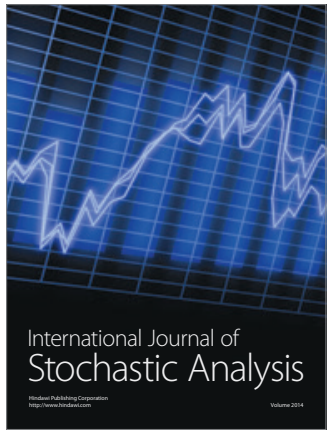

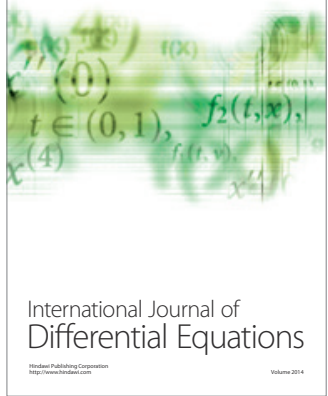
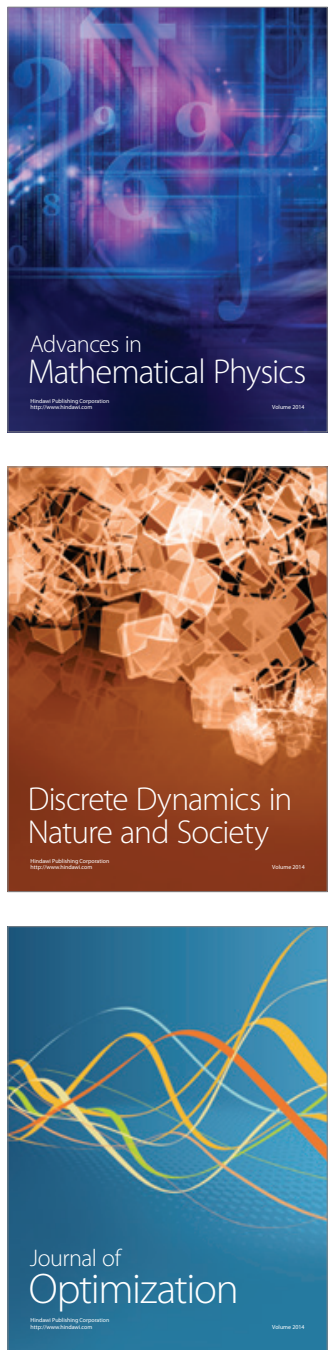